Volume 8. No. 9, September 2020

International Journal of Emerging Trends in Engineering Research

Available Online at http://www.warse.org/IJETER/static/pdf/file/ijeter133892020.pdf

https://doi.org/10.30534/ijeter/2020/133892020

\title{
The Patient Organism Modeling for Diagnosis with the Usage of a Multi Agent Representation
}

\author{
Oleksandr Kuzomin ${ }^{1}$, Oleksandra Dudka ${ }^{2}$, Oleksii Vasylenko ${ }^{3}$, Vyacheslav Lyashenko ${ }^{4}$ \\ ${ }^{1}$ Department of Informatics, Kharkiv National University of RadioElectronics, Ukraine, \\ a.oleksandr.kuzomin@gmail.com \\ ${ }^{2}$ Department of Radio Technologies Information and Communication Systems, Kharkiv National University of \\ RadioElectronics, Ukraine, oleksandra.dudka@nure.ua \\ ${ }^{3}$ Manager of Architecture for the Big Data and Analytics department, Toyota corp., Sydney, Australia, \\ b.ichbinerste@gmail.com \\ ${ }^{4}$ Department of Informatics, Kharkiv National University of RadioElectronics, Ukraine, \\ lyashenko.vyacheslav@gmail.com
}

\begin{abstract}
The main ideas for building a multi-agent model of intelligent modeling for diagnosis are offered. The peculiarities of actions in a certain system of medical environment are indicated. The function of a medical agent is defined, which determines the actions that can be used by a medical agent in response to any sequence of acts of perception of medical data and knowledge. The problem of development of the specification of the problem medical agent is considered. The direction of development of the program of the medical agent is shown. Consider the requirements for the creation of reflex medical agents that respond directly to acts of perception. The requirements for the design of target-based agents are also considered.
\end{abstract}

Key words: Medical Agent, Multi-Agent Model, Intelligent Medical Diagnostic System, Artificial Intelligence

\section{INTRODUCTION}

Problems of human disease medical diagnosis are very difficult. But now there is an opportunity to use the achievements of artificial intelligence (AI) to obtain a timely and better diagnosis of human disease [1]-[5]. It is through the use of models based on multi-agent representation that we have the opportunity to formulate a qualitative paradigm for building a model of medical thinking for diagnosis.

Therefore, considering the problem from the system approach to the analysis for the diagnosis purpose, it is necessary to perform the distribution of the main purpose of diagnosis process. To obtain the most reasonable treatment scenario through a systematic analysis of the human condition. Data on the human condition involve the implementation of preliminary diagnosis on the basis of clinical tests (analysis of blood, urine, etc.).

To solve the stated problem, we use the concept of a rational medical agent, which is central in this study. The concept of rationality that can be to a wide variety of medical agents applied that can be used in diagnosis. In any environment that can only be imagined representing the internal state of the patient through the use in addition to laboratory and clinical tests, the results of the study of internal organs, as well as the environment that directly affects the human condition.

It is possible to identify the data and knowledge used to diagnose pain in more detail and reliably according to the purpose of diagnosis is to use this concept to develop a small set of system design principles to create successful medical agents - that is, a system that could well be called intelligent diagnostic agents [6].

\section{USE OF MULTI AGENTS FOR DESIGN OF DIAGNOSTIC MEDICAL SYSTEM}

The aim of diagnosis of human disease is to solve significant problems, which currently have many theoretical and practical successes. Currently, different areas of medicine use specific to this area diagnostic methods.

For example, in general surgery, the following methods are used [6]-[8]:

- external examination (as a rule, the general appearance of the patient is examined: color and structure of integuments, mucous membranes, places of a source of pain, etc.);

- biopsy - examination under a microscope (histological examination) of a biopsy (tissue sample taken from a living organism); 
Oleksandr Kuzomin et al., International Journal of Emerging Trends in Engineering Research, 8(9), September 2020, 5733 - 5739

- laparoscopy - examination of the abdominal cavity with a special chamber, which is inserted into the abdominal cavity through an incision about 1-1.5 centimeters wide;

- research with the help of probes, a special camera (including the gastrointestinal tract);

- palpation (used, as a rule, for the initial determination of closed fractures and bone tavma, the primary diagnosis of some surgical syndromes);

- radiography (usually in traumatology and pulmonology);

- ultrasound and other studies using radiation technologies.

Laboratory diagnostics is a set of methods aimed at the analysis of the studied material with the help of various specialized equipment. Moreover, at the first stage of diagnosis there is communication between the doctor and the patient due to the communication of the patient with the doctor (the anamnesis is revealed).

Laboratory methods (blood, urine, feces, biopsy, etc.), as well as instrumental methods (ECG, ultrasound, X-ray, endoscopic, etc.) in the model can be represented by individual agents.

Regarding the diagnosis from a systematic approach, it should be borne in mind that the above-mentioned features of the diagnosis should be taken into account when modeling the condition of the patient's body, as a multi-agent representation. But here we must take into account the problem, which is based on:

- individual approach to each patient,

- the ability to take into account when diagnosing multi-vector analysis of medical data and knowledge (using radiation studies) cannot make it possible to obtain an objective diagnosis in minimal time, with minimal costs and not too effective on patients analysis results that have devastating clinical and psychological impact on patient (too much medical data and knowledge does not always give a positive result).

Therefore, it is important to choose a sufficiently effective set of tests and studies for each patient and the possibilities of a systematic approach to the design of medical information system (MIS) using a multi-agent representation of a set of intelligent agents for the problem of diagnosing the patient. Various medical technologies and technical means are involved in the diagnostic process. They provide a comprehensive and detailed view. But to combine all the information, it is necessary to use computer, information, medical diagnostic technologies and the use of artificial intelligence technologies. That is, to implement a systematic approach to the design of medical intelligent diagnostic system (MIDS).

Summarizing the above, it is necessary to use AI to perform modeling of the human body, its disease, the medical environment and in general on the basis of intelligent solutions to obtain a diagnosis with minimal risk of medical error in a short time with minimal money.

Therefore, it is from these positions that it is expedient to use multi-agent representation of technical means, algorithms and software tools in modeling.

It is known that a multi-agent system (MAS) is a system formed by several interacting intelligent agents that sell a variety of medical devices. Multi-agent systems can be used to solve problems that are difficult or impossible to solve with a single agent or a monolithic system [9].

Then the patient's organism model (MOP) can be represented as:

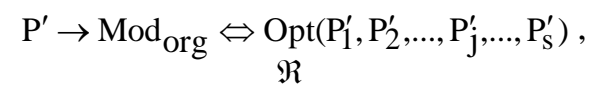

where $\mathrm{P}^{\prime}=\left\{\mathrm{P}_{1}^{\prime}, \mathrm{P}_{2}^{\prime}, \ldots, \mathrm{P}_{8}^{\prime}\right\}$ is the a set of statistical procedures for the transformation of information about MOP; $\mathrm{P}_{1}^{\prime}-$ procedure of multifactor analysis and ranking of MOP parameters; $\mathrm{P}_{2}^{\prime}$ - procedure of regression analysis of statistical or ontological data of symptoms and signs; $\mathrm{P}_{3}^{\prime}-$ procedure of analysis of variance of symptoms and signs; $\mathrm{P}_{4}^{\prime}-$ the procedure for identifying the diagnosis of the victim with the highest probability of reliability; - procedure of cluster analysis of the subject area; $\mathrm{P}_{5}^{\prime}$ - Data Mining procedure; $\mathrm{P}_{6}^{\prime}$ - compiling a decision tree; $\mathrm{P}_{7}^{\prime}$ - procedure using fuzzy logic of neural networks; $\mathrm{P}_{8}^{\prime}$ - procedure for combining the results of systematic analysis of ontology data and MOP precedents and analysis of patients' condition EDR, Internet search, data discovery, knowledge of textual information about multiple diagnoses $\left\{\mathrm{Si}_{\mathrm{D}} \mathrm{O}\right\}$, symptoms $\{\mathrm{Si}\}$ and signs $\{\mathrm{O}\}$ of desyфs and logical laws of clinical thinking; $\mathrm{P}_{9}^{\prime}-$ procedure for assessing the risk of misdiagnosis in patients with sets $\{\mathrm{D}\},\{\mathrm{Si}\},\{\mathrm{O}\} ; \mathrm{P}_{10}^{\prime}$ - procedure for determining a clinical diagnosis using multiple "microsituations" $\left\{\mathrm{Si}^{\mathrm{Msi}} \mathrm{O}\right\}$.

As a result of research on the results of preliminary analysis of a priori data of the subject area of MOP to find the diagnosis, symptom, diagnostic sign, we have a generalized model of 
MOP taking into account the logical connection of the set of symptoms $\{\mathrm{Si}\}$, the set of signs $\{\mathrm{O}\}$ and microsituations $\left\{\mathrm{Si}^{\mathrm{Msi}} \mathrm{O}\right\}$ variants of the diagnosis of the disease $\left\{\mathrm{Si}_{\mathrm{D}} \mathrm{O}\right\}$ :

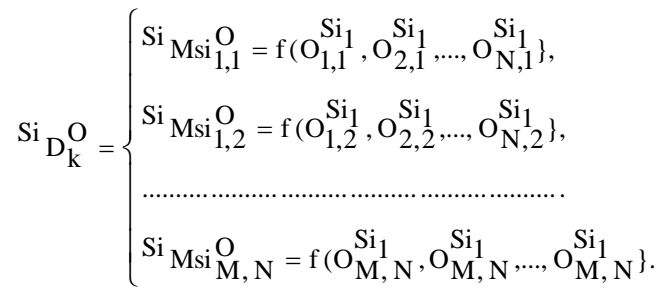

where $\mathrm{k}=\overline{1, \mathrm{M}}, \mathrm{d}=\overline{1, \mathrm{~N}}$.

Consider the features of the use of agents in MAS.

\section{AGENTS AND OPTIONS OF PRESENTATION OF DATA ON THE CONDITION OF HUMAN BEINGS AND THE ENVIRONMENT}

For a person who will be diagnosed, it is possible to use the model as a multi-agent system with data on the internal elements of the human body (body temperature, test results, computed tomography studies, etc.).

On the Figure 1 the following is presented:

Agent Type - Intelligent Medical Diagnostic Agent (IMDA).

Environment - Patient, hospital, staff.

Executing mechanisms - Derivation of questions, tests, diagnoses, medical recommendations, directions for research.

Sensors - Entering from the keyboard data on symptoms in accordance with the international symptoms classification ISC 10, the results of laboratory clinical studies, conclusions on the analysis of images of elements of the human body after CT, MRI, etc.

For systematic analysis of data and knowledge about IMAD, it is necessary to determine what the performance indicators of the agent are.

Input data from sensors or sensors, which can also be keys when, after pressing the keys, the data enters the Intelligent Medical Diagnostic Agent (IMDA) software. The software displays data, writes files and transmits them to network packets for analytical presentation. In addition, it is sometimes possible to take into account the general assumption that each agent may perceive its own therapeutic actions (but not always their results).
Here it is possible to use the term perception to denote the received IMDA sensory data at any particular time. The sequence of acts of perception by the agent is the complete medical history of everything that was ever perceived by the medical agent (Figure 1).

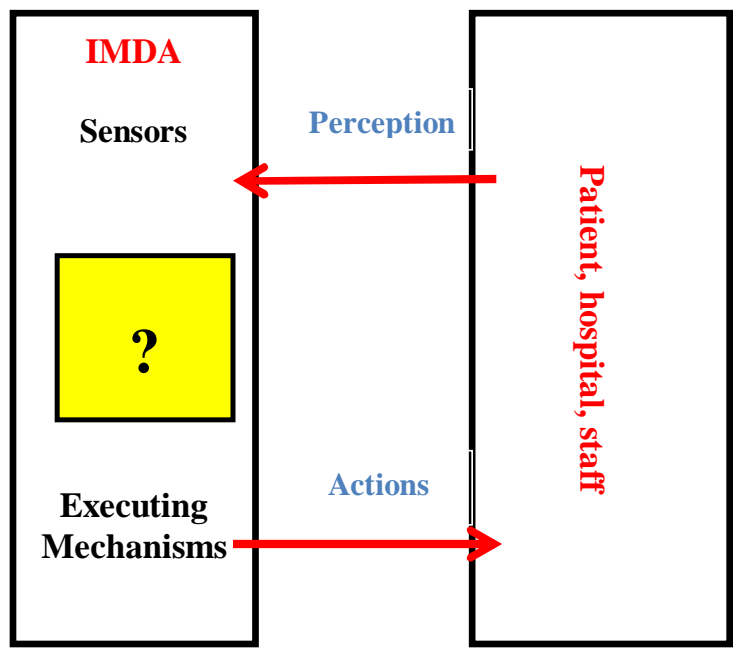

Figure 1: IMDA interacts with the environment through sensors and actuators

Generally speaking, the choice of action by a medical agent at any given time may depend on the entire sequence of acts of perception observed up to that point in time. If it is possible to determine which effect will be chosen by IMDA in response to any possible sequence of acts of perception, it is possible to have a more or less accurate definition of IMDA. From a mathematical point of view, this is tantamount to claiming that the behavior of a medical agent can be described using the IMDA function, which reflects any particular sequence of acts of perception on an action.

The task of tabulating the function of a medical agent, which describes any particular agent, may be considered; for most agents, this would be a very large table (actually infinite) if the length of the sequences of acts of perception, which must be taken into account in the table of data and knowledge about the disease, is not set. If IMDA uses a certain randomization of data to select its actions, it may be necessary to check each sequence repeatedly to determine the probability of each medical action. Such an organization of the IMDA can be very intelligent.

Such a table is certainly an external description of a medical agent. The internal description is to determine which agent functions for a given artificial agent is implemented using the agent program. It is important to distinguish between the last two concepts. The agent function is an abstract mathematical description, and the medical agent program is a specific implementation operating within the medical agent architecture. 
Oleksandr Kuzomin et al., International Journal of Emerging Trends in Engineering Research, 8(9), September 2020, 5733 - 5739

\section{QUALITATIVE BEHAVIOR AND THE CONCEPT OF RATIONALITY}

A rational diagnostic agent is an agent that performs the correct diagnostic actions. In the first approximation we can say that the correct medical action is the action that ensures the most successful functioning of IMDA in diagnosis and treatment. Therefore, a certain way of measuring success in achieving the goal of diagnosis is needed. Success criteria, along with a description of the environment (Figure 1), as well as sensors and actuators IMDA, provide a complete specification of the task faced by the agent. With these components, we can define more precisely what is meant by the word "rational".

Consider the performance indicators of IMDA, which embody the criteria for assessing the successful behavior of a medical agent. After immersion in the medical environment, the medical agent produces a sequence of medical actions that correspond to the received perception. This sequence of actions forces the environment to go through a sequence of states. If such a sequence corresponds to the desired success in diagnosing the disease, then IMDA functions well in accordance with the goal of diagnosis. Of course, there cannot be one constant indicator suitable for all medical agents.

Therefore, it is necessary to persistently seek the use of objective indicators of IMDA performance, and, as a rule, the designer who designs IMDA provides such indicators. As a general rule, it should be noted that it is better to develop performance indicators according to what really needs to be achieved in this treatment environment, rather than according to how, in the opinion of the designer, the medical agent should behave.

The task of choosing performance indicators is not always easy.

- At any given time, the assessment of the rationality of IMDA's actions depends on the following four factors:

- IMDA performance indicators that determine the criteria for successful diagnosis.

- Agent's knowledge of previously acquired environmental and medical environment, i.e. ontological data [9].

- Therapeutic actions that can be performed by a medical agent.

- The sequence of acts of perception by the medical agent that have taken place so far.

Taking into account these factors, the following definition of a medical rational agent can be formulated.

For each possible sequence of perceptions, the rational medical agent must choose a medical action that is expected to maximize his performance of diagnosis and treatment, taking into accounts the facts provided by this sequence of perceptions and all built-in medical knowledge possessed by the medical agent.

It is easy to find that in other circumstances the same agent may become irrational.

\section{AWARENESS, LEARNING AND AUTONOMY}

The distinction between rationality and omniscience must be made carefully. The omniscient medical agent knows the actual result of his actions and can act accordingly; but omniscience is in fact impossible. Rationality is the maximization of expected productivity, and perfection is the maximization of the actual productivity of diagnosis and treatment. In renouncing the pursuit of perfection, we not only apply fair criteria to agents, but also take into account reality. The fact is that if an agent is required to perform the actions that prove best after their implementation, the task of designing an agent that meets this specification becomes impossible (in particular, until we can increase the efficiency of time machines or crystal balls used by fortune tellers).

Therefore, our definition of rationality does not require omniscience, because rational choice depends only on the sequence of acts of perception, formed to date.

Our definition requires that the rational agent not only gather information, but also learn as much as possible from the data he perceives. The initial configuration of the agent may reflect some prior knowledge of the environment, but as the agent gains experience, this knowledge may be modified and supplemented. There are extreme cases in which the environment is fully known in advance. In such cases, the agent does not need to perceive information or learn; it just works right away. Of course, such agents are very vulnerable. successful agents, the task of calculating the function of the agent is divided into three separate periods: when designing an agent, some calculations are performed by its designers; the agent performs additional calculations by selecting one of his next actions; and as the agent learns from experience, he performs other auxiliary calculations to decide how to modify his behavior.

If the degree, to which the agent relies on the a priori knowledge of its designer, rather than on their perceptions, is too high, then such an agent is considered to have insufficient autonomy. A rational agent must be autonomous - he must learn everything he can to compensate for incomplete or incorrect a priori knowledge.

It should be noted that an important source for providing the agent with additional information is related to the use of ontologies and precedents in the design [9], [10]. 
From the point of view of practice, the agent is often required to be completely autonomous from the beginning: if the medical agent has little or no experience, he is forced to act randomly if the designer has not provided him with some assistance. Therefore, just as evolution has given animals enough innate reflexes to live after birth long enough to learn on their own, it would be wise to give an artificial intelligence agent some initial knowledge, not just give him the ability to learn. After sufficient experience of existence in the environment the behavior of the rational medical agent can become essentially independent of his a priori knowledge. Therefore, the inclusion in the project of the ability to learn allows you to design simple rational medical agents that can operate successfully in a wide variety of environments.

\section{GOAL BASED AGENTS}

Knowledge of the current state of the medical environment is not always sufficient to decide what to do when diagnosing. The medical agent needs not only a description of the person's current condition, but also some information about the purpose of diagnosing the disease, which describes the desired situation, the diagnosis and treatment of the person's condition. The medical agent program may combine this information with information about the results of possible medical actions (with the same information as that used to update the internal state of the reflex medical agent) to select medical actions to achieve this goal of successful diagnosis. The structure of the target-acting medical agent is shown in Figure 2.

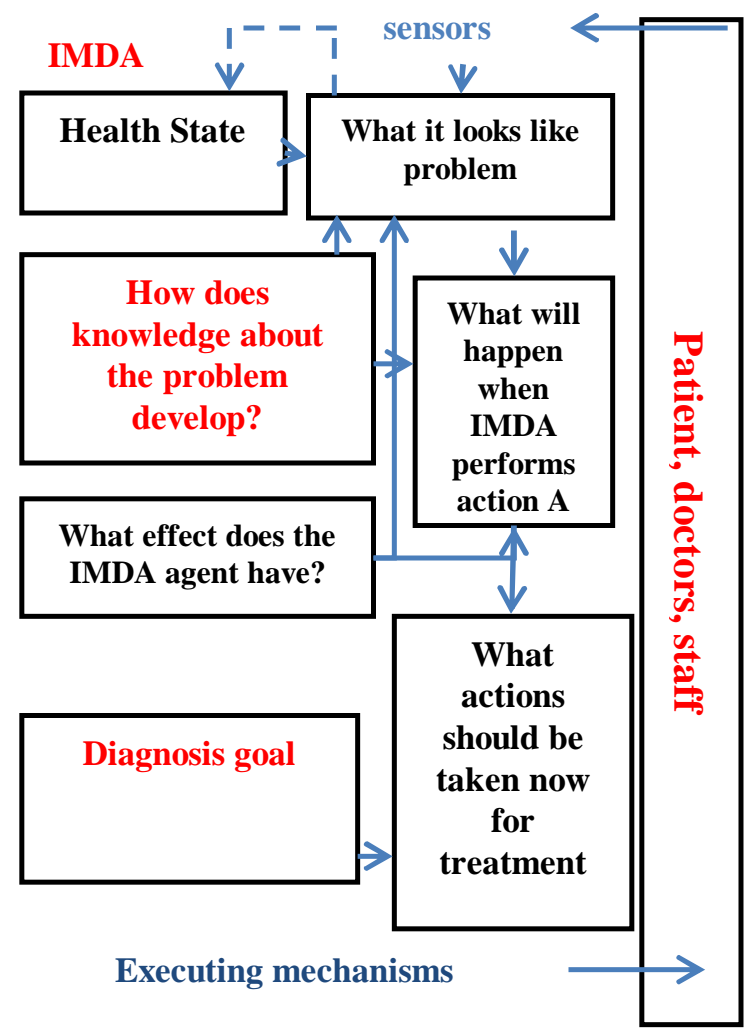

Figure 2: Agent based on the goal and on the model
Sometimes the task of choosing a medical action based on the goal of diagnosis and treatment is solved simply when the goal is immediately the result of a single action of treatment, and sometimes the task becomes more difficult and the medical agent needs to consider long sequences of corrections to find a way to achieve the goal of diagnosis. Subsectors of artificial intelligence, which are dedicated to developing sequences of actions that allow a medical agent to achieve its goal, are the search and planning of treatment solutions.

An agent based on the model (3) and on the goals monitors the state of knowledge about the treatment direction, as well as the number of goals that he tries to achieve in the diagnosis and chooses the action that allows (ultimately) achieving these goals.

It should be borne in mind that this kind of decision-making procedure has fundamental differences from the above-described procedure of application of the conditions-action rules, because it has to think about the future, answering two questions: "What will happen if I do this and that? "and" Will this allow me to have fun? "In the projects of medical reflex agents such information is not presented explicitly, because the built-in rules establish a direct correspondence between perceptions and actions.

Given the principle that usually changes the medical world, for him the only action that allows to achieve such a goal as the prevention of inaccurate or erroneous diagnoses.

Although at first glance it seems that a goal-based medical agent is less effective, it is more flexible because the knowledge on which its decisions are based is clearly presented and can be modified, for example, by information from the Internet, i.e. online data knowledge.

For a reflex medical agent, on the other hand, in this case would have to rewrite a number of rules "condition-action".

The behavior of the medical agent based on the goal can be easily changed to direct it to another place of search, and the rules of the reflex mobile agent, which indicate where the return and where treatment can be performed in the same "direction" as before, before, will be applicable only to a single destination; in order for this agent to be sent to another location, all of these rules must be replaced.

\section{UTILITY BASED AGENTS}

In fact, in most environments, accounting for goals alone is not enough to develop high-quality behavior.

The goals allow only a hard-binary distinction between states of "satisfaction" and "dissatisfaction", while more general 
performance indicators should provide a comparison of different states of the medical world in exact accordance with how satisfied IMDA will be if they can be achieved. Because the concept of "satisfaction" is not entirely scientific, more commonly used terminology, according to which the state of the world, defined in more detail than others, is considered to have a higher utility for the agent. The term "utility" means "property to be useful", and not a secondary action that has secondary services [9].

The utility function maps a state (or sequence of states) to a real number, which indicates the appropriate degree of satisfaction of the medical agent.

The complete specification of the utility function provides the ability to make rational decisions in the two cases described below, when the goals do not allow it. First, if there are conflicting goals, such that only some of them can be achieved (for example, speed or safety), the utility function allows you to find an acceptable compromise.

Second, if there are several goals that an agent can pursue, but none of them can be achieved with all certainty, the utility provides a convenient way to weigh the probability of success, taking into account the importance of goals.

Any rational agent should behave as if it had a utility function, the expected value of which it tries to maximize.

Therefore, an agent with a clearly defined utility function has the ability to make rational decisions and is able to do so using a general-purpose algorithm that does not depend on the specific utility function that is maximized.

Due to this view, the "global" definition of rationality (according to which the functions of the medical agent with the highest productivity are considered rational) turns into a "local" constraint on the projects of rational medical agents, which can be expressed as a simple program.

The structure of the agent, acting on the basis of utility, is shown in Figure 3.

It uses the model of the medical world along with the utility function, which measures the agent's preferences over the medical world. The agent then selects an action that leads to the best expected utility.

To calculate the expected utility, averaging is performed for all possible resultant states, taking into account the coefficient that determines the probability of each result.

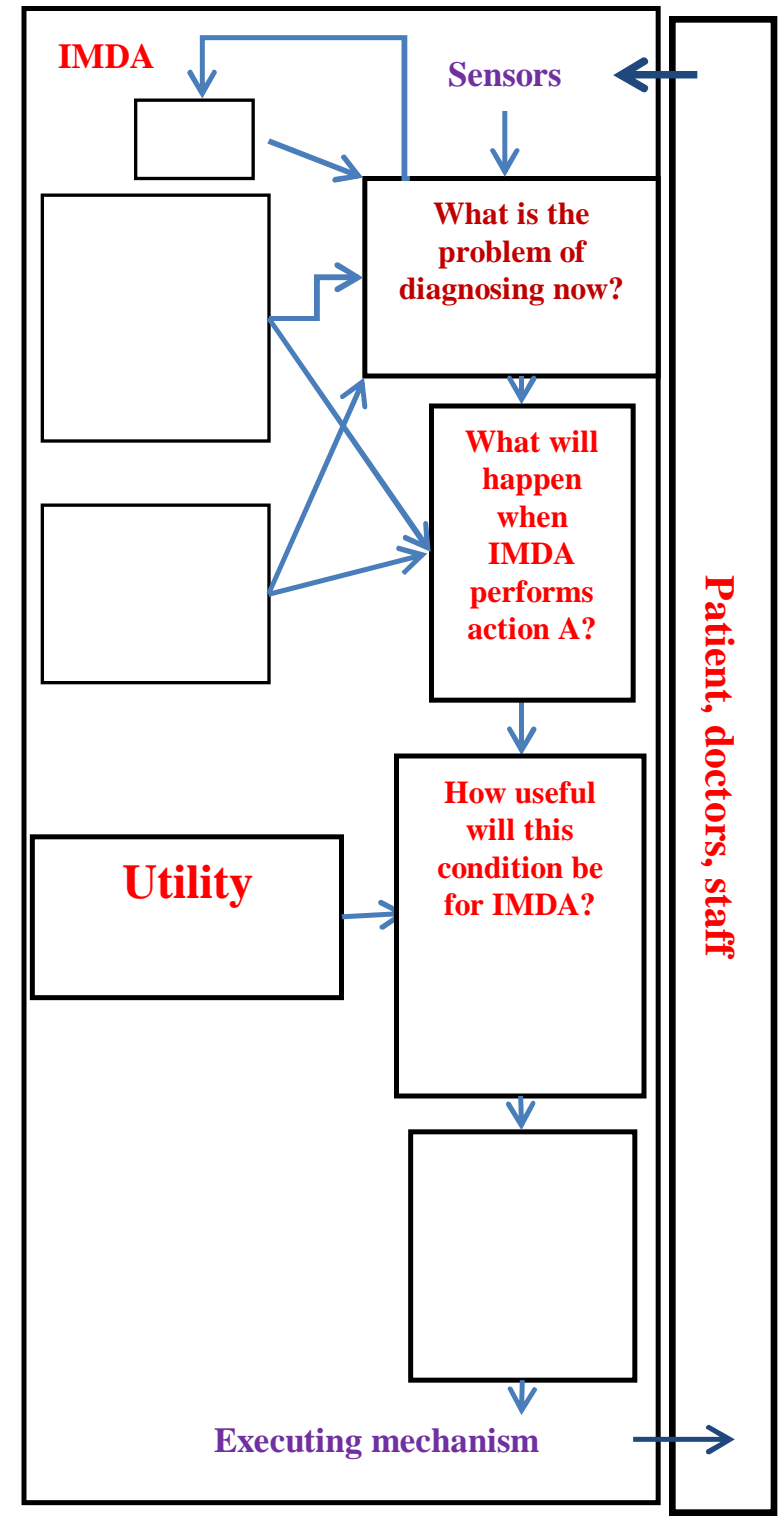

Figure 3: Utility based agent

The programs of medical agents in which various methods of a choice of medical actions are applied were described above.

\section{CONCLUSION}

In this study, the basic ideas for building the IMDA model were examined. First, it is proposed to use the concept of diagnostic micro-situation, which is the result of classification and clustering of medical data on the situation with the human condition, which has developed as a result of preliminary analysis of ontological and precedent knowledge. Secondly, the main means of modeling the organism using multi-agent representation are shown. 
Oleksandr Kuzomin et al., International Journal of Emerging Trends in Engineering Research, 8(9), September 2020, 5733 - 5739

\section{REFERENCES}

1. F. Hidayanti, H. H. Santoso, and H. E. Prasetyo. Upper Stomach Disorder Detection System using Backpropagation Artificial Neural Network, International Journal of Emerging Trends in Engineering Research, Vol. 8, no. 8, pp. 4426-4432, 2020. https://doi.org/10.30534/ijeter/2020/62882020

2. P. Orobinskyi, D. Petrenko, and V. Lyashenko. Novel Approach to Computer-Aided Detection of Lung Nodules of Difficult Location with Use of Multifactorial Models and Deep Neural Networks, in 15th International Conference on the Experience of Designing and Application of CAD Systems (CADSM), pp. 1-5, 2019.

https://doi.org/10.1109/CADSM.2019.8779340

3. A. M. Babker, A. E. A. Altoum, I. Tvoroshenko, and V. Lyashenko. Information technologies of the processing of the spaces of the states of a complex biophysical object in the intellectual medical system health, International Journal of Advanced Trends in Computer Science and Engineering, Vol. 8, no 6, pp. 3221-3227, 2019.

https://doi.org/10.30534/ijatcse/2019/89862019

4. S. Sotnik, R. Matarneh, and V. Lyashenko. System Model Tooling For Injection Molding, International Journal of Mechanical Engineering and Technology, Vol. 8, no. 9, pp. 378-390, 2016.

5. M. Anila, and G. Pradeepini. Diagnosis of Parkinson's Disease using Artificial Neural Network, International Journal of Emerging Trends in Engineering Research, Vol. 8, no. 7, pp. 3700-3707, 2020.

https://doi.org/10.30534/ijeter/2020/131872020

6. S. Russell, and P. Norvig. Artificial intelligence: a modern approach, Prentice Hall, New Jersey, 2002.

7. J. Shen, and et al.. Artificial intelligence versus clinicians in disease diagnosis: Systematic review, JMIR medical informatics, Vol. 7, no. 3, pp. e10010, 2019. https://doi.org/10.2196/10010

8. K. H. Yu, A. L. Beam, and I. S. Kohane. Artificial intelligence in healthcare, Nature biomedical engineering, Vol. 2, no. 10, pp. 719-731, 2018. https://doi.org/10.1038/s41551-018-0305-z

9. O. Kuzomin, O. Dudka, O. Vasylenko, V. Radchenko, and V. Lyashenko. Using of Ontologies for Building Databases and Knowledge Bases for Consequences Management of Emergency, International Journal of Advanced Trends in Computer Science and Engineering, Vol. 9, no. 4, pp. 5040-5045, 2020. https://doi.org/10.30534/ijatcse/2020/123942020

10. I. Tvoroshenko, M. A. Ahmad, S. K. Mustafa, V. Lyashenko, and A. R. Alharbi. Modification of Models Intensive Development Ontologies by Fuzzy Logic, International Journal of Emerging Trends in
Engineering Research, Vol. 8, no. 3, pp. 939-944, 2020. https://doi.org/10.30534/ijeter/2020/50832020 